\title{
MOVE-IT: DEVELOPMENT AND EVALUATION OF EFFICIENT TRAINING PROCE- DURES FOR ELDERLY ROAD USERS TO SUPPORT THEIR DRIVING COMPETENCE
}

\author{
Stefanie Schoch ${ }^{1}$, Ruth Julier ${ }^{1}$, Ramona Kenntner-Mabiala ${ }^{1}$, Yvonne Kaussner ${ }^{1}$ \\ ${ }^{1}$ Würzburg Institute for Traffic Sciences (WIVW), Veitshöchheim, Germany \\ Email: schoch@wivw.de
}

\begin{abstract}
Summary: Evaluated training measures for improvement of elderly drivers' driving competency lead to a considerable better driving performance, but so far are time-consuming and costly, making its nationwide implementation difficult. The aim of the present project was to develop and evaluate a modular training program for elderly drivers, which is easy, low-cost and time efficient. Based on an individual profile including driving related performance deficits and the individual need for mobility, a personalized training program is compiled. It includes individualized driving exercises, group sessions to refresh knowledge of traffic rules, consultation regarding the compensation of age-related restrictions and the use of driver assistance systems. For evaluation, a pre-post-design with 30 subjects was realized. The driving performance was measured through different performance parameters and increased through participation. Driving instructors rated the program useful and feasible. Participants were very satisfied with the concept of the training and evaluated it as being helpful. Therefore, this training concept seems to be promising for future use beyond the project work. A scientifically accompanied introduction of the training concept to several driving schools is recommended.
\end{abstract}

\section{INTRODUCTION}

Driving is of great importance for the elderly as it enables the maintenance of individual mobility, compensates the decreasing physical mobility and is therefore an important resource for autonomous living. However, increasing age makes the occurrence of driving related impairments (e.g. failing eyesight, cognitive slowing, physical limitations, chronic illness; e.g. Anstey et al., 2003) more probable, with a significant inter-individual heterogeneity having to be taken into account. Scientific studies have shown that training can sustain and even expand cognitive function throughout the ageing process. The largest success of training can be seen in skills that were trained directly (Poschadel, 2013). This means that in order to enable individual mobility by car as long as possible, the driving task itself has to be practiced. Accordingly, several studies have shown that elderly drivers can benefit from specific driver education even at old age (Korner-Bitensky et al., 2009; Kaussner \& Kenntner-Mabiala, 2015). Sawula et al. (2018) showed that individualized driver training reduced $30 \%$ in overall number of driving errors in comparison to participants in a basic in-class training group, Lavallière et al. (2012) showed that simulator training combined with driving-specific feedbacks helped older drivers to improve their visual inspection strategies, whereas Romoser et al. (2012) showed that the reduction of unacceptable driving behavior after post-drive feedback following a simulated drive could be maintained over two years. Although there are some evaluated training programs that lead to substantial increases in performance of the elderly driver, conducting those programs often is both very time-consuming and costly (e.g. Cassutt et al., 2014), making its nationwide implementation difficult. Other training programs that are offered in Germany often are not evaluated, do not include on the road driving 
training, do not consider the elderly drivers' special needs or heterogeneity or rather have the diagnostic aim of testing the fitness to drive than actual training. The study by Coxon et al. (2016) even shows the importance of chosen content and methodology for gaining positive training effects, as the program did not result in behavior modification, but in increased depression scores for the group that had participated in the training sessions.

This project uses previous findings both on existing training programs and identification of mobility characteristics and mobility sub-groups to develop and evaluate a short and extensively applicable training program which can be conducted by driving instructors independently. Due to its modular concept it can easily be personalized to the driver's specific needs. Elderly drivers with driving-related performance impairments should be trained in a manner that they can master all driving tasks. At the same time, if drivers are no longer able to or do no longer want to drive on certain road types, due to the adaptability of the training concept, they do not have to practice these specific driving tasks. Thus, a high efficiency of the training program is ensured. By offering such a training program, individual mobility of elderly drivers is supposed to be maintained, while at the same time general traffic safety can be increased. The training concept was evaluated by a pre-post-design, using both ratings of driving competency under real driving conditions and subjective ratings as criteria for the success of the training. The leading research questions were (1) if the training program improves driving performance of elderly, impaired drivers (effectiveness on concept level); (2) if all of the training modules are useful (effectiveness on modular level); (3) if the training program is perceived as useful by driving instructors and participants.

\section{METHOD}

\section{The Training Concept}

The SOK-model from Baltes et al. (1998), previously used by different relevant study groups for the implementation of training, was the basis for the development of this concept. The general model of successful development implies that a successful adaptation to age-related cognitive and bodily changes can be achieved through three strategies: selection, optimization, and compensation. The theory assumes that a selection of goals or functional areas (e.g. specifying and choosing goals, rearranging one's goal hierarchy) becomes necessary due to a limitation of resources within the person. Selected resources are acquired or developed through the process of optimization (e.g. practice or acquiring new abilities). To maintain a functional level a loss of goal-relevant resources must be compensated (e.g. using external help or substituting resources). Transferring the SOK-model to the development of a training concept for elderly road users, the strategies can be applied as follows: Selection of drives, routes or conditions which should generally be avoided by the elderly driver due to his performance profile or which he is ready to avoid (e.g. unknown routes, highway, etc.); Optimization through advisement and training (e.g. driving lessons focused on relevant topics for the individual senior driver); Compensation through the application of strategies (e.g. driving with navigation system, avoiding driving at night). Thus, selection and compensation can make the driving task easier, whereas optimization aims at maintaining and increasing driving competence by specific practice. 
The training concept MOVE-IT is made up of three parts (Figure 1): The anamnesis with an analysis of mobility needs and performance diagnostics; the training with measures adapted to individual driving performance and mobility needs, and an evaluative final assessment.

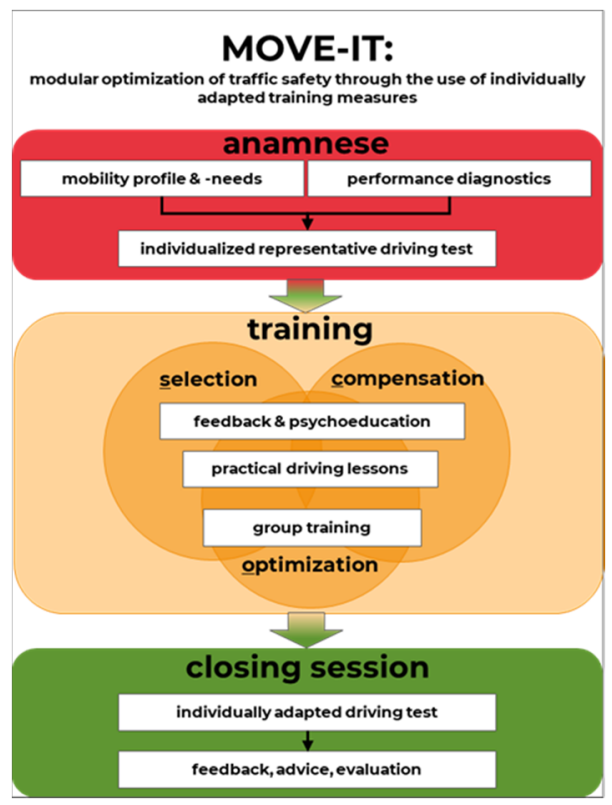

Figure 1. The training concept MOVE-IT adapted from the SOK model of Baltes et al. (1998)

The anamnesis offers insight into the mobility profile, the need for mobility, and cognitive performance of the individual. Through an individualized but representative driving performance test under real driving conditions it is tested if the person can reach the mobility goal. This means that the driving test has to contain all relevant driving tasks that might occur to a driver due to the personal mobility profile. If any discrepancies between driving performance and mobility goal become apparent, further measures can be defined using a personalized training plan. The training should target those areas where deficits have become apparent, and if necessary and feasible offer concrete and practical practice. Different modules can be chosen to treat specific deficits (Figure 2).

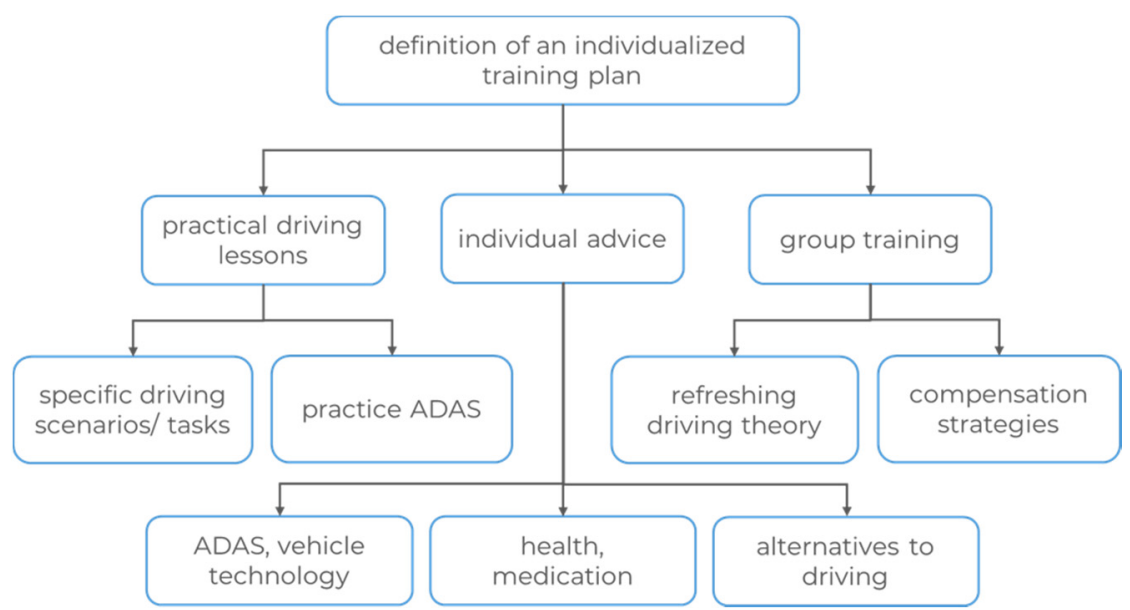

Figure 2. The different training modules which can be selected and adaptively combined as needed 
The main goal is the improvement of driving performance of the elderly driver and the transfer of knowledge to sensitize for the consequences of performance deficits. At the end a second driving performance test is conducted to evaluate the learning success. The program was designed to be held by trained driving instructors. Elaborate material has been developed to support both participants and driving instructors. The time frame of this program is demand-oriented. Participants without discrepancies between their performance diagnostic and their mobility goal have the lowest time cost, as they only need the performance diagnostic and the feedback discussion, which take two sessions and approximately 2.5 hours. Participants who require a lot of training and need all modules have the largest time cost with approximately 10 hours and 7 sessions.

\section{Experimental Designs}

Subjects. For the evaluation of the training program, elderly road users of at least 75 years, who are active drivers but have doubts concerning their fitness to drive were recruited using advertisements and mouth-to-mouth propaganda. Overall, 63 seniors were interested in participating. Of these, 55 seniors met the inclusion criteria. 46 persons completed the anamnesis, with 13 seniors not showing any conspicuous driving behavior and thus having to be excluded from the evaluation study. Three persons withdraw from the study: One person due to health issues, one person due to concerns regarding legal consequences of conspicuous driving behavior, and one person with a very critical driving performance did not fulfil the driving instructor's requirements to first have the field of vision tested before continuing. At the end of the study data of 30 fully trained participants was analyzed $(\mathrm{m}=78.57$ years; $\mathrm{sd}=5.57 ; 11$ females). Although all of the participants reported health issues, most of them rated their health condition to be "good" considering their age $(\mathrm{m}=2.27 ; \mathrm{sd}=0.64 ; 1=$ very good; $6=$ very poor $)$. Main motivation for participation was to receive an objective feedback on their ability to still drive safely.

Procedures. During the anamnesis all participants were asked about their current mobility behavior and their mobility goals by means of a semi-structured interview. Furthermore, participants took a vision test, psychometric performance test and a theoretical test concerning traffic knowledge. Subsequently, the participants completed a driving performance test in the driving instructor's dual-control car under real driving conditions. Performance was assessed by a driving instructor and a blinded psychologist with regard to different driving parameters. It contained all individually relevant driving tasks, e.g. driving on rural roads, highways or in complex cities, turning, priority rules, and took about 45 to 60 minutes. All participants received feedback and recommendations for future measures after the driving test. Participants who showed no or slightly conspicuous driving behavior (rating of 4 or less on a 11-point fitness-to-drive scale: 0-3 normal behavior, 4-6 conspicuous behavior, 7-10 precarious behavior) and did not need further measures besides receiving feedback from the driving instructor, completed an evaluation form but were excluded from the evaluation study. For the remaining participants, an individual training plan based on the anamnesis was developed and conducted. This could contain up to 3 personalized driving lessons with a driving instructor, including counseling on specific topics; group sessions with regard to traffic knowledge taking approximately 1.5 hours; and selection of driving tasks, e.g. suggesting not to drive on certain road types any more.

After completion of the training program the driving performance test was repeated. The pre-post design was chosen as the individually adaptive manner of the program can hardly be tested with 
a control group design. As the driving instructors also had conducted the training program, it was assumed that they would not be fully objective with regard to driving performance. Thus, it was of importance that the accompanying psychologist, who assessed the driving performance on the objective level, did not know anything about the training concept. The driving instructors' and the participants' ratings were used to evaluate perceived usefulness and feasibility for everyday life. Further distribution of the training program beyond this project work largely relies on the acceptance of participants and driving instructors.

Evaluation. The evaluation was conducted on two levels: the concept- and modular level. It was the aim of this project to evaluate the efficiency of both the overall training concept and the training blocks. The comparison of driving performance during the anamnestic and the final driving assessment is the basis for the evaluation. Overall driving competency was rated by a driving instructor, the driver and a blinded psychologist. Additionally, to give detailed feedback about which aspects of driving competency had been improved throughout the training, different types of driving errors (cognitive, speed, lane keeping, precarious behavior) were registered and classified by the psychologist using a tablet-application for real traffic in both drives. In order to evaluate the perceived usefulness of the training, both participants and driving instructors completed questionnaires on general and modular acceptance and satisfaction. On modular level three training blocks were evaluated separately: driving lessons with a driving instructor including consultation, selection of driving tasks and a group session regarding traffic knowledge. For evaluation the frequency of the driving errors associated with the focus of the driving lessons, as well as the results of the theoretical tests concerning traffic knowledge pre and post training were compared. Moreover, the questionnaires concerning perceived usefulness and acceptance of each of the training blocks were analyzed.

\section{RESULTS}

Results of 30 participants were analyzed. For comparison of pre and post driving performance ttests for dependent samples were used. During the anamnestic drive the psychological experimenter rated the mean driving performance with $\mathrm{m}=5.50, \mathrm{sd}=1.358$ (fitness to drive scale: $0=$ normal behavior; $10=$ precarious behavior), corresponding conspicuous driving behavior. After completion of the program the mean rating of driving competency by the experimenter was significantly lower $(\mathrm{m}=3.93, \mathrm{sd}=1.363 ; \mathrm{t}(29)=5.32 ; \mathrm{p}<.001, \mathrm{~d}=1.151$; Figure 3$)$, matching normal to slightly conspicuous driving. The mean of driving competency rated by the driving instructor during the anamnestic drive was $\mathrm{m}=6.03, \mathrm{sd}=0.999$, which signifies conspicuous driving behavior. After completion of the training the driving instructor rated the driving performance significantly better, i.e. normal to slightly conspicuous $(\mathrm{m}=3.70, \mathrm{sd}=1.149 ; \mathrm{t}(29)=10.30, \mathrm{p}<.001, \mathrm{~d}=$ 2.162). Correspondingly, driving errors occurred significantly less after having completed the training program (overall number of driving errors: $\mathrm{t}(29)=8.11 ; \mathrm{p}<.001$ ). This applies especially for the typical cognitive driving errors, such as not looking over the shoulder, not indicating, hesitant lane changing. Other typical driving errors which could be significantly reduced were driving too fast or with a headway distance being too small, and poor lane keeping. Therefore, the training showed a large positive effect on the overall improvement of driving competency on the concept level. In general, the participants were very satisfied with the program. The duration of the program was rated as being appropriate. Most participants would be willing to pay between 20 and $90 €$ per session $(\mathrm{m}=44.63 ; \mathrm{sd}=14.21)$ for such a program in the future. 

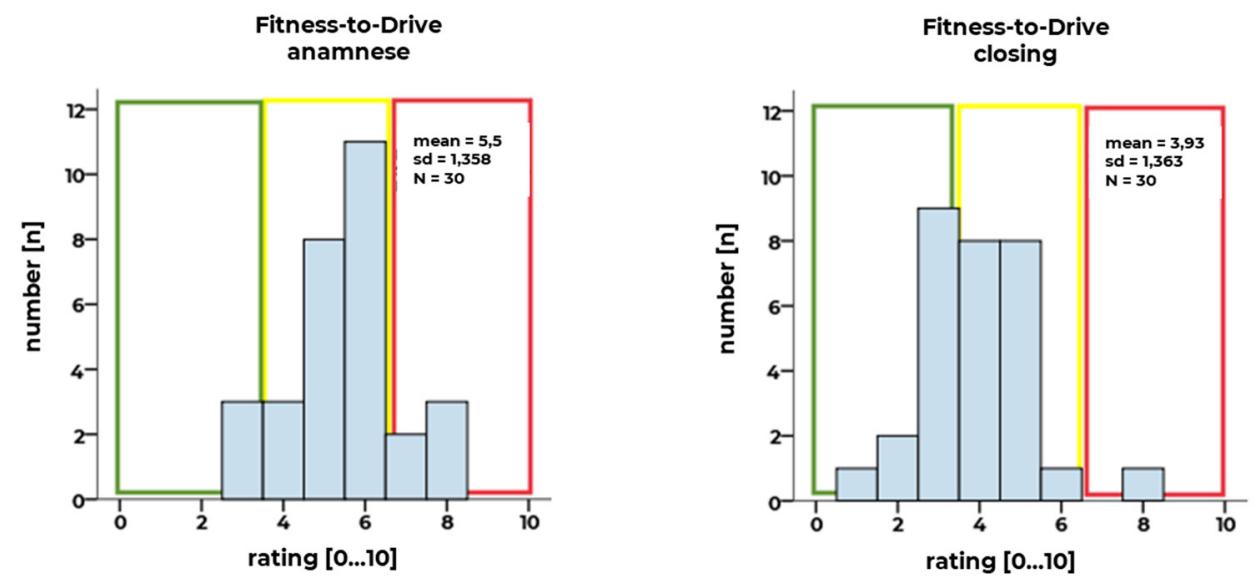

Figure 3: Driving competence rating of the psychologist during amnestic and closing drive

The evaluation on the modular level showed a positive effect for all modules. The effectiveness of the training block driving lesson can be seen in the theme- and situation specific analysis of the driving errors and competency ratings: specific driving errors decreased with targeted training. For example, an increase in driving competency in urban areas could be seen if drives in the city had been practiced. Correspondingly, drivers looked more often and earlier before changing lanes or turning after having practiced the behavior during driving lessons. An overall increase of driving competency could be seen by the driving instructor from driving lesson to driving lesson $(F(2)=26.14 ; p=.000)$. Acceptance for this module was rated as very high by the participants. The individual consultation was rated as useful. The group sessions regarding traffic knowledge lead to an improvement in a traffic knowledge test compiled of official test questions (post-training vs. pre-training) and was rated as useful by the participants.

\section{CONCLUSIONS}

The evaluation with a small sample of 30 seniors showed that the training concept is effective and a promising approach for future use beyond the project work. Each of the training blocks proved to be useful and accepted by both driving instructor and participant. Thus, the training concept might be helpful to maintain the elderly driver's mobility as long as possible and to increase general traffic safety. However, based on the small number of persons that participated in this program, generalizations should be kept to a minimum. Therefore, the authors propose implementing the concept under scientific supervision at several driving schools nationwide. The main question should be whether the results of this study can be generalized for other driving schools, a larger sample, other regions and other routes. Additionally, investigating long-term effects of the training on the driving performance of the elderly road user would be of interest. Furthermore, marketing strategies and information campaigns should be developed to increase attraction and reach the target group.

\section{ACKNOWLEDGMENTS}

This report is based on parts of the research project carried out at the request of the Federal Ministry of Transport and Digital Infrastructure, represented by the Federal Highway Research Institute, under research project No. $82.0650 / 2016$. The author is solely responsible for the content. 


\section{REFERENCES}

Anstey, K. J., Hofer, S. M., \& Luszcz, M. A. (2003). A latent growth curve analysis of late-life sensory and cognitive function over 8 years: evidence for specific and common factors underlying change. Psychol. Ageing 18, 714-726.

Baltes, M. M., Lang, F. R., \& Wilms, H.-U. (1998). Selektive Optimierung von Kompensation: Erfolgreiches Altern in der Alltagsgestaltung. In A. Kruse et al. (Ed.), Psychosoziale Gerontologie, Band 1, Grundlagen, 188-202. Göttingen: Hogrefe.

Cassutt, G., Theill, N., Martin, M., \& Jäncke, L. (2014). The Drive-Wise Project: Driving Simulator Training increases real driving performance in healthy older drivers. Frontiers in Aging Neuroscience, 6, 85.

Coxon, K., Chevalier, A., Brown, J. \& Keay, L. (2016). Effects of a Safe Transportation Educational Program for Older Drivers on Driving Exposure and Community Participation: A Randomized Controlled Trial. Journal of American Geriatrics Society, 60, 540-549.

Kaussner, Y., \& Kenntner-Mabiala, R. (2015). MobilTrain: Erhaltung und Erweiterung von Kompetenzen zur Förderung der Individualmobilität von Senioren. Würzburg, DOI: $10.2314 / \mathrm{GBV}: 866224416$.

Korner-Bitensky, N., Kua, A., Von Zweck, C., Van Benthe, K. (2009). Older Driver Retraining: An Updated Systematic Review of Evidence of Effectiveness. Journal of Safety Research, 40, 105-111.

Lavallière, M., Simoneau, M., Tremblay, M., Laurendeau, D., \& Teasdale, N. (2012). Active training and driving-specific feedback improve older drivers' visual search prior to lane changes. BMC Geriatrics 12(5), doi:10.1186/1471-2318-12-5.

Poschadel, S. (2013). Trainierbarkeit der Fahrkompetenz im Alter. In B. Schlag \& K. J. Beckmann (Ed.), Mobilität und demografische Entwicklung, 181-210. Köln: TÜV Media $\mathrm{GmbH}$.

Romoser, M.R.E., (2012). The Long-Term Effects of Active Training Strategies on Improving Older Drivers' Scanning in Intersections: A Two-Year Follow-Up to Romoser and Fisher (2009). Human Factors: The Journal of the Human Factors and Ergonomics Society, 55(2), 278-284.

Sawula, E., Polgar, J., Porter, M.M., Gagnon, S., Weaver, B., Nakagawa, S., Stinchcombe, A., \& Bédard, M. (2018). The combined effects of on-road and simulator training with feedback on older drivers' on-road performance: Evidence from a randomized-controlled trial. Traffic Injury Prevention, 19(3), 241-249. 